\title{
EXTRAOCULAR MUSCLE PROBLEMS IN THYROID EYE DISEASE
}

\author{
PETER FELLS $^{1}$, LINDA KOUSOULIDES $^{1}$, ANASTASIA PAPPA ${ }^{1,2}$, \\ PETER MUNRO $^{2}$ and JOANNA LAWSON ${ }^{1}$ \\ London
}

\begin{abstract}
SUMMARY
In this paper methods of visualisation of the extraocular muscle changes in thyroid eye disease are discussed. The histopathology of extraocular muscle biopsies has been studied by both light and electron microscopy to show the type of cellular infiltration and the amorphous material in the extracellular matrix. A series of questions to which answers have not yet been found concerning thyroid eye disease are posed which may help to direct new research projects. Finally, in the last part of the paper, the surgical results in a series of $\mathbf{4 1}$ patients having ocular muscle surgery for diplopia and/or compensatory head postures due to thyroid eye disease are described. The conclusions drawn from these results are that one should maintain the patient euthyroid, establish by orthoptic measurements that the ocular movements have been stable for at least 6 months, treat by recessing tight muscles using adjustable sutures, and aim to undercorrect the vertical deviation at the time of adjustment.
\end{abstract}

Once post-mortem examinations of the orbits of patients with thyroid eye disease had revealed the enormous swelling of the extraocular muscles it was gradually realised that the muscular changes may be responsible for many of the symptoms and signs in this condition. The first part of this paper will review ways in which the extraocular muscles can be investigated. The second part will pose some of the many questions that need to be answered in this condition. Finally, the results of surgical intervention to correct some of the consequences of these pathological changes in the muscles are discussed.

\section{INVESTIGATION OF THE EXTRAOCULAR MUSCLES}

\section{Visualisation of Extraocular Muscle Changes}

Surgical exploration of gross unilateral proptosis to

From: 'Moorfields Eye Hospital, London; ${ }^{2}$ Institute of Ophthalmology, London, UK.

Correspondence to: Peter Fells, FRCS, Moorfields Eye Hospital, City Road, London EC1V 2PD, UK. achieve a diagnosis was necessary before special scanning techniques became available. The huge muscles in a patient with $13 \mathrm{~mm}$ of unilateral proptosis but no other mass in any quadrant confirmed the diagnosis of thyroid eye disease and also established the fact that a difference of more than $6 \mathrm{~mm}$ proptosis between the two eyes did not mean some cause other than thyroid eye disease as had previously been accepted.

Computed tomography $(C T)$ scans $^{1}$ at first gave axial views of the orbits; then direct coronal slices could be taken with the patient's neck hyperextended in the largediameter port of the whole body scanner (which was not possible with the dedicated head and neck scanners). Reformatted coronal views are used now, together with reformatted views along the optic nerve axis. Proptosis is the first evidence of auto-decompression, followed by outward bowing of the medial wall encroaching into the ethmoidal space which makes the 'Coca-cola bottle' sign when it occurs bilaterally.

Magnetic resonance imaging (MRI) can give even more information about the muscle enlargement without any radiation risk. The enviable ease of repeated views to assess response to therapy is offset by their high cost in some parts of the country.

Ultrasonography in B-scan mode can show the size of some of the rectus muscles in the anterior part of their course. A-scan mode has been used to assess ocular muscle reflectivity, with echogenicity lower than $40 \%$ suggesting that a better response to therapy with immunosuppressives may be expected. ${ }^{2}$

\section{Histopathology of Extraocular Muscle Biopsies}

Extraocular muscle biopsies from patients with thyroid eye disease were examined by means of immunohistochemistry and light and electron microscopy. The biopsies were taken under local anaesthesia from the belly of the involved recti muscles, often during the early stages of the disease before ocular rotations were reduced and before 


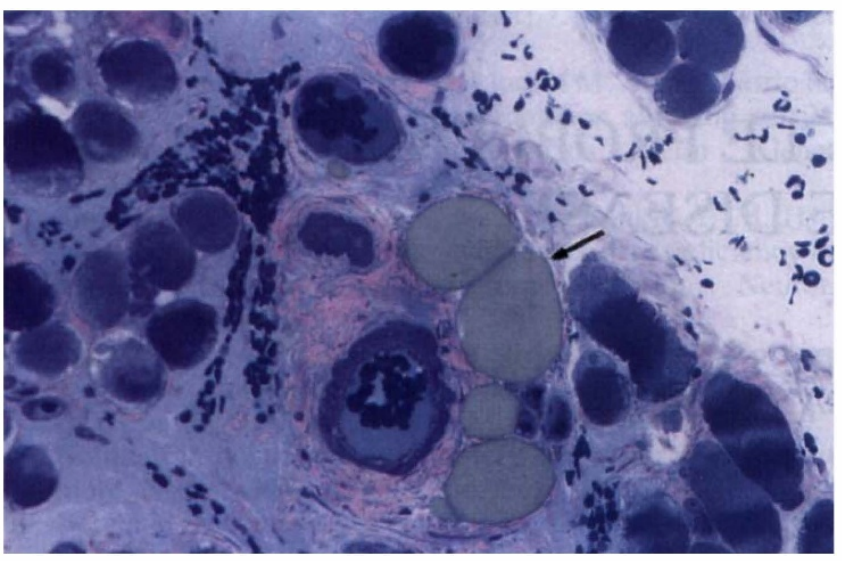

Fig. 1. Light micrograph of transversely cut extraocular muscle tissue from a patient with thyroid eye disease. Staining with toluidine bluelbasic fuchsin on a resin section shows increased interfibrillar spaces, fatty cell infiltration (arrow) as well as increased numbers of collagen fibres denoted by the red colour. $(\times 750)$

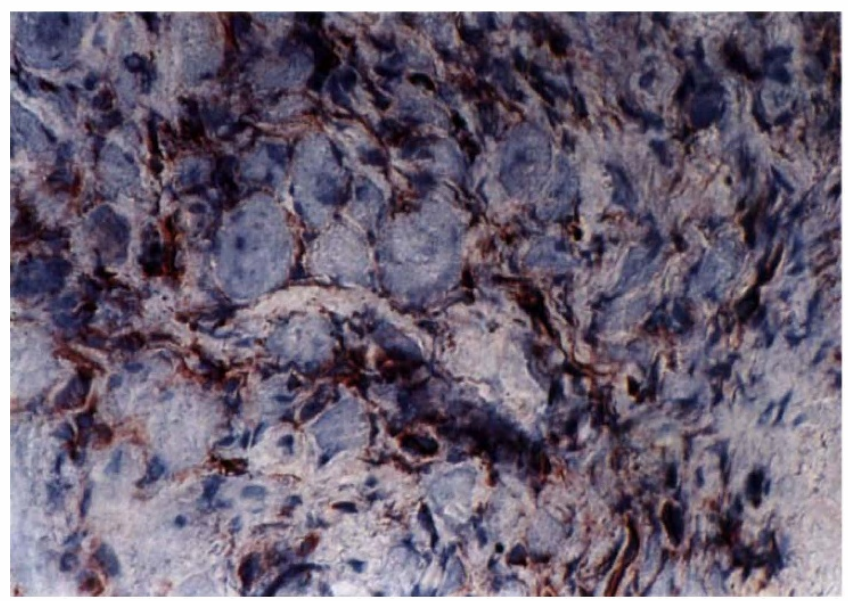

Fig. 3. Light micrograph of immunoperoxidase staining for intracellular adhesion molecule 1 (ICAM-1) on a cryostat section of extraocular muscle tissue from a patient with thyroid eye disease. ICAM-1 expression is seen as a brown precipitate. $(\times 750)$

immunosuppressive therapy was started. The inferior and medial recti were the most commonly biopsied muscles.

Microscopic examination demonstrates either focal or diffuse infiltration of the spaces between the extraocular muscle fibres with mononuclear inflammatory cells including lymphocytes, macrophages and mast cells (Figs. 1,2). The interfibrillar spaces were enlarged and contained an amorphous material in the extracellular matrix. This amorphous material has been shown immunohistochemically to have hyaluronic acid as a major component, using a recently developed monoclonal antibody against hyaluronic acid.

Infiltration of the endomysial space by lymphocytes, macrophages and neutrophils is observed during the early stages of the disease. These cells decrease in numbers as the disease burns itself out and are replaced by collagen

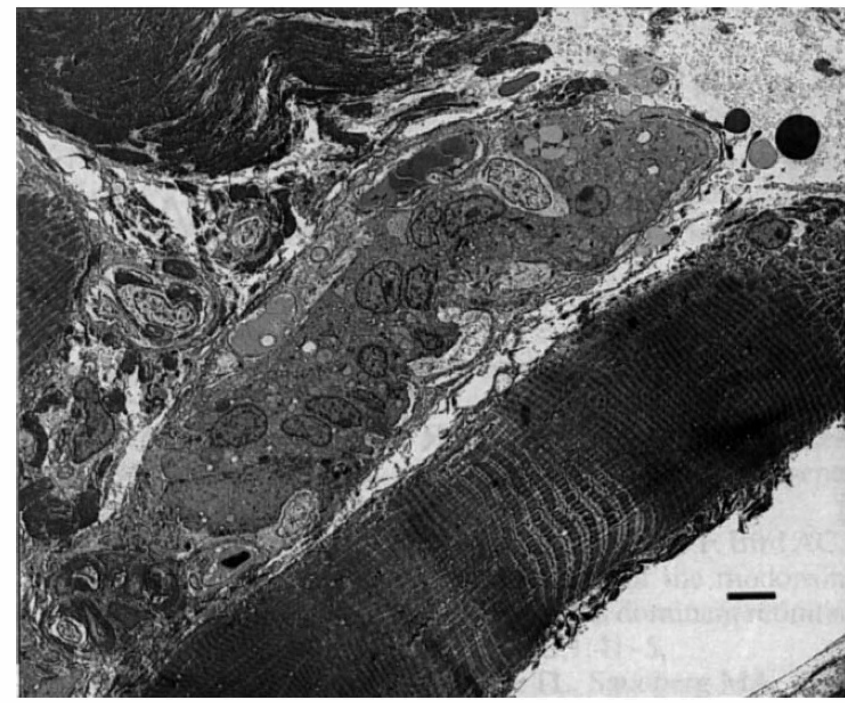

Fig. 2. Transmission electron micrograph illustrating a perivenular inflammatory cell infiltrate in association with a longitudinally cut extraocular muscle fibre of a thyroid eye disease, extraocular muscle biopsy. Scale bar represents $5 \mu m(\times 2200)$

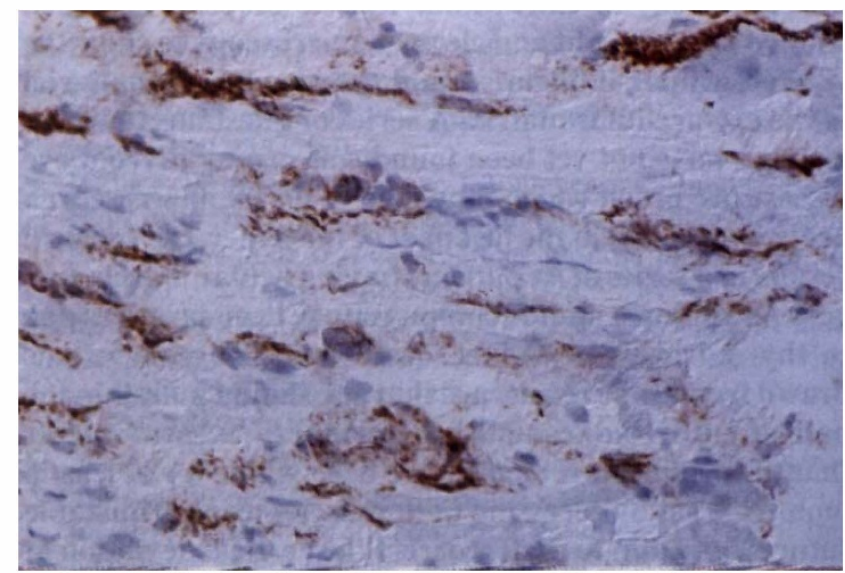

Fig. 4. Light micrograph of immunoperoxidase staining for HLA class II (DR) on a cryostat section of extraocular muscle tissue from a patient with thyroid eye disease. Positively stained cells and their processes are located in the endomysium and perimysium (brown precipitate). $(\times 476)$

and fat cells, the presence of which adversely affects the contractile properties of the affected muscle.

Lymphocytic infiltration coincides with upregulation of adhesion molecules ${ }^{3}$ on the surface of both lymphocytes and vascular endothelium (Fig. 3). Increased expression of HLA class II molecules can be detected by means of immunohistochemistry on the surface of the cells from the endomysium and perimysium, probably fibroblasts (Fig. 4). Some of the infiltrating lymphocytes are activated and produce lymphokines which either attract other inflammatory cells into the area or stimulate fibroblasts. ${ }^{46}$ Stimulated fibroblasts in turn produce increased quantities of glycosaminoglycans (linear polysaccharide molecules) which osmotically attract water causing interstitial oedema. ${ }^{7}$ The fibroblasts are also more numerous in the connective tissue. Muscle fibres themselves are thought 
not to be affected and to be similar to control fibres, but morphometric measurements of muscle fibre area and diameters have yet to be completed.

\section{Muscle Activity Analysis}

Muscle activity can be analysed by means of (1) lengthtension curves of extraocular muscles, (2) electromyography (EMG) and (3) intraocular pressure changes in certain gaze directions.

\section{Orthoptic Testing}

Orthoptic testing can be done using (1) Hess charts, (2) field of binocular single vision (BSV) and (3) uniocular fields of fixation. We first used this last method in 1969 but abandoned it because the results were inconsistent and provided no more useful information than the Hess charts and BSV fields elicited. We tried again in 1991/2 but with similar lack of success and no longer use that method.

The methods we find most useful in clinical practice are CT scans, serial Hess charts and fields of BSV.

\section{QUESTIONS IN THYROID EYE DISEASE}

Some major questions in thyroid eye disease are:

Are the signs of optic neuropathy due to direct compression of the optic nerve by enlarged muscles at the orbital apex?

How many signs of thyroid eye disease can be attributed to orbital venous stasis from vein compression (a thesis developed by Feldon)?

How can clinically unilateral proptosis occur?

Why are not all the extraocular muscles enlarged equally?

Are the oblique muscles involved?

How can the proptosis of the second eye be delayed, sometimes for many years, and still be due to thyroid eye disease?

Why do some patients have slight proptosis and signs of severe optic nerve compression?

Why do some patients have marked proptosis and no signs of optic nerve compression?

How do some patients have marked proptosis and no signs of extraocular muscle enlargement?

Are the reduced ocular rotations always due to tight muscles restricting movement?

Does ocular muscle paresis occur as well?

When is the right time to operate on the extraocular muscles?

How does botulinum toxin injection work in thyroid eye disease?

How can botulinum toxin give long-lasting improvement?

We have partial answers to a few of these questions. Reduced ocular movements are normally due to tethering by tight rectus muscles, the inferior and medial recti being most often involved. However, careful performance of the traction test under general anaesthetic may reveal less res- triction than expected. Conversely in some patients with a positive traction test, i.e. decreased movement, rotation may not return to normal when the appropriate rectus muscle has been fully detached during surgery.

Botulinum injections within a year of onset of restricted movements can give much improved movement. ${ }^{8}$ This cannot be affecting fibrous tissue but must be working on the muscles to relax the active contraction that Simonsz and Kommerell ${ }^{9}$ have shown. Unfortunately the rapid change from hypo- to hypertropia after inferior rectus toxin upsets many patients, so we no longer use it.

After surgical orbital decompression, ${ }^{10}$ which for many surgeons means using the ethmoidal space and the antral sinus medial to the infraorbital nerve, patients often show increased esotropia, usually with an A-pattern. When the orbital resistance has been reduced the tight medial recti may pull the eyes into esotropia. Esotropia may be increased by tight inferior recti too. The A-pattern results from disinsertion of the inferior oblique muscles when the medial part of orbital floor is removed, and the reduced proptosis also encourages increased superior oblique action.

The timing and sequence of surgery to the extraocular muscles is most important. ${ }^{11}$ If orbital decompression is indicated it must be done first. Even when decompression is not required in the absence of optic neuropathy or severe corneal exposure, if proptosis is greater than $26 \mathrm{~mm}$ then recessing tight recti can make the proptosis worse. Hence proptosis of $26 \mathrm{~mm}$ and more should be reduced before ocular muscle surgery. Next, ocular movement stability is essential before trying to correct diplopia. In the past this would mean waiting 2 years before ocular muscle surgery. We use stable ocular measurements on the Hess chart and BSV fields for at least 6 months as our criteria. Continuing active orbital inflammation needs to be identified and avoided. Mourits developed an index of clinical activity using the long-established criteria for inflammation of pain, redness, swelling and impaired function. We have found this difficult to use, in particular when attempting to characterise the different types of orbital pain, although the changes in oedema are helpful. Other objective methods of assessing active inflammation include using ultrasonography in A-scan and B-scan modes, and the different $T$ spins in MRI scanning. Kahaly and, independently, one of the present authors (A.P.) have demonstrated increased urinary glycosaminoglycans (mucopolysaccharides) in the active phase of orbital inflammation.

\section{SURGICAL RESULTS}

A series of 41 patients underwent ocular muscle surgery for diplopia and/or compensatory head posture due to thyroid eye disease. Twenty-eight were female and 13 male, of whom 25 were cigarette smokers and 7 had other autoimmune diseases such as pernicious anaemia and vitiligo. Thirty patients were hyperthyroid initially, 6 hypothyroid and 5 euthyroid, although all had been rendered euthyroid by the time of their strabismus surgery. Thirteen had been treated by radioactive iodine, 2 by partial thyroidectomy 


\section{Group 1: Excellent Outcome}

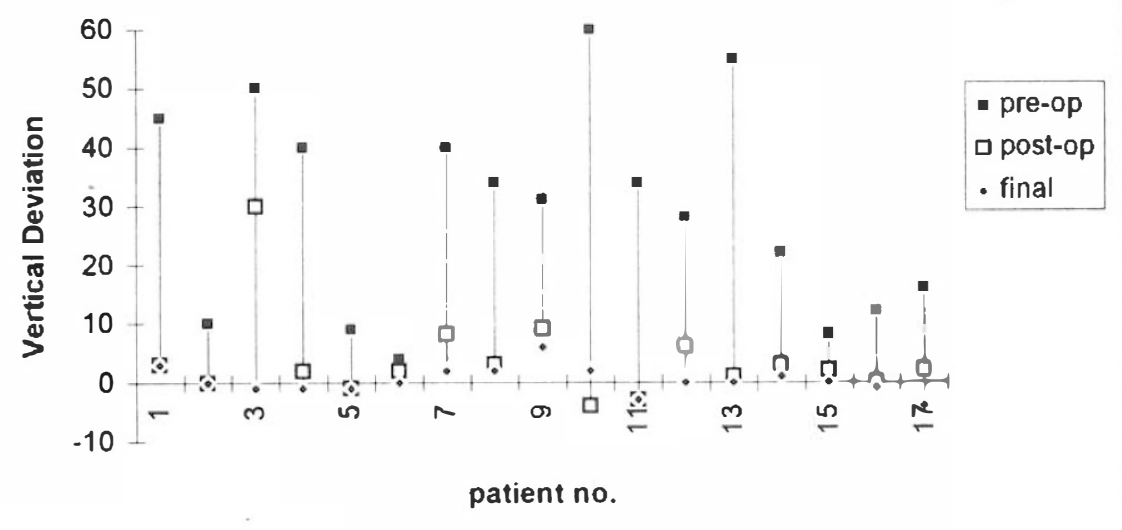

(a)

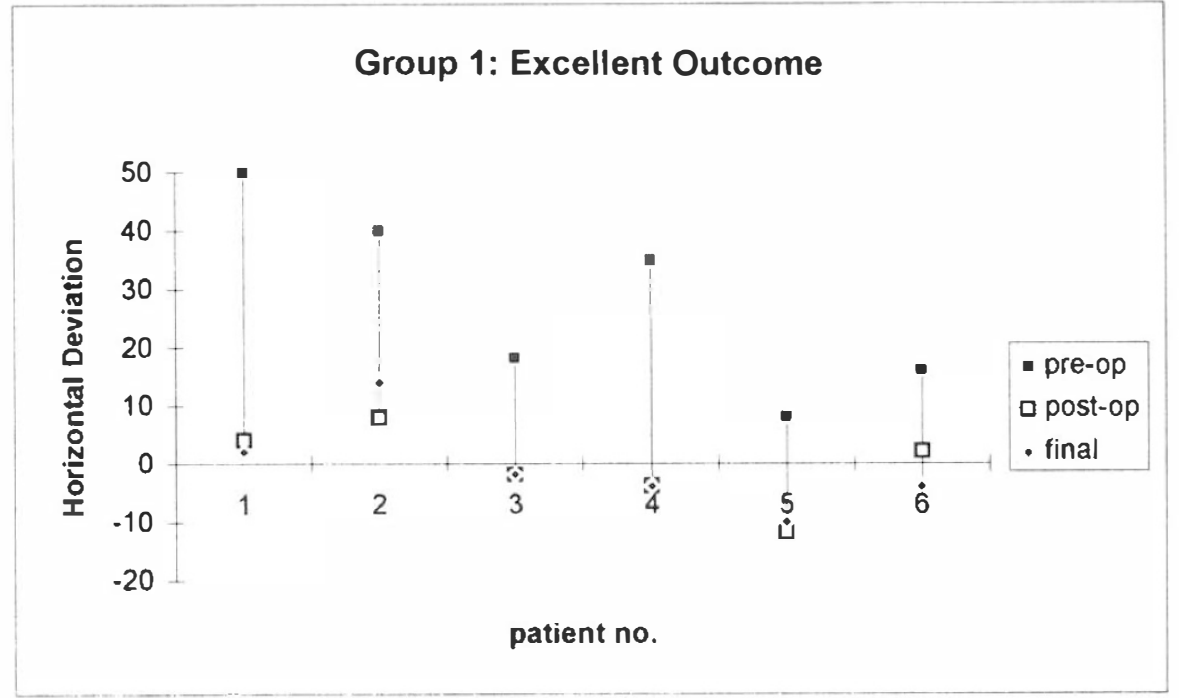

(b)

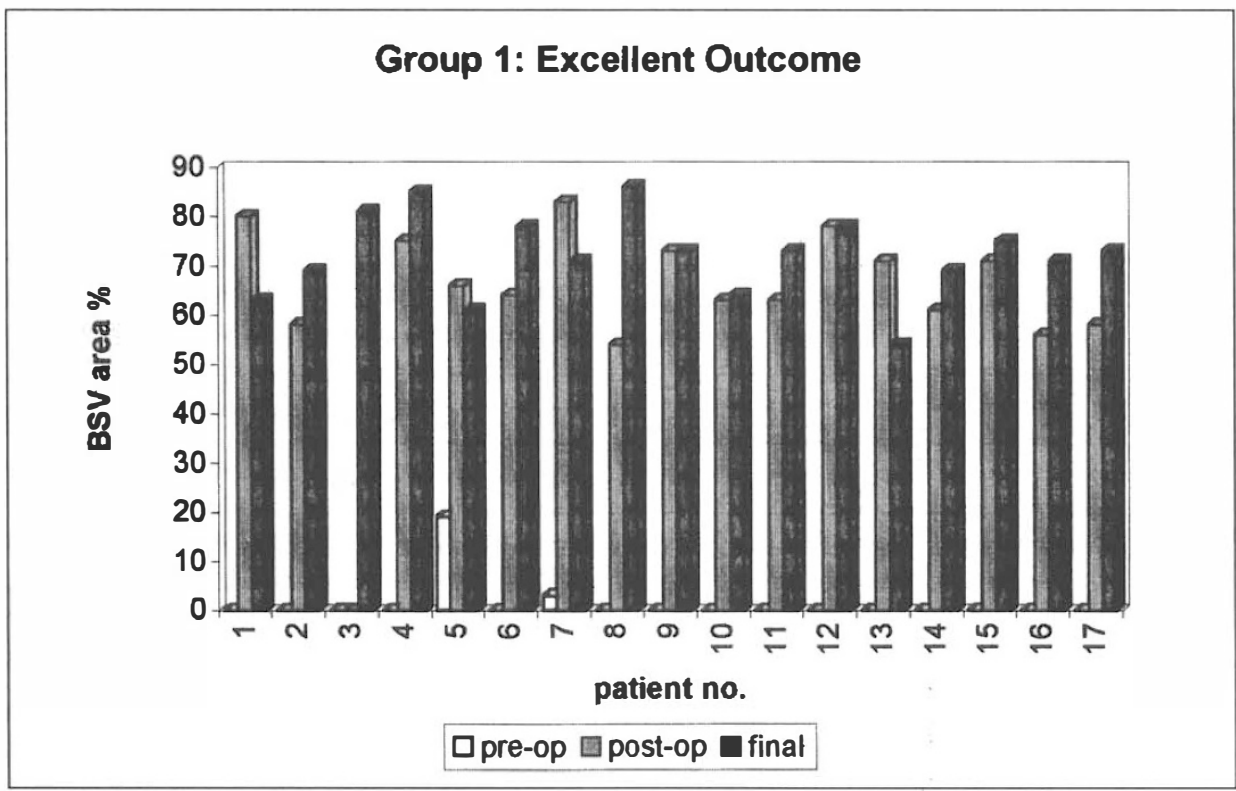

(c)

Fig. 5. Results of the excellent outcome group (group 1) showing the improvement in the vertical deviation, horizontal deviation and area of binocular single vision following surgery. 


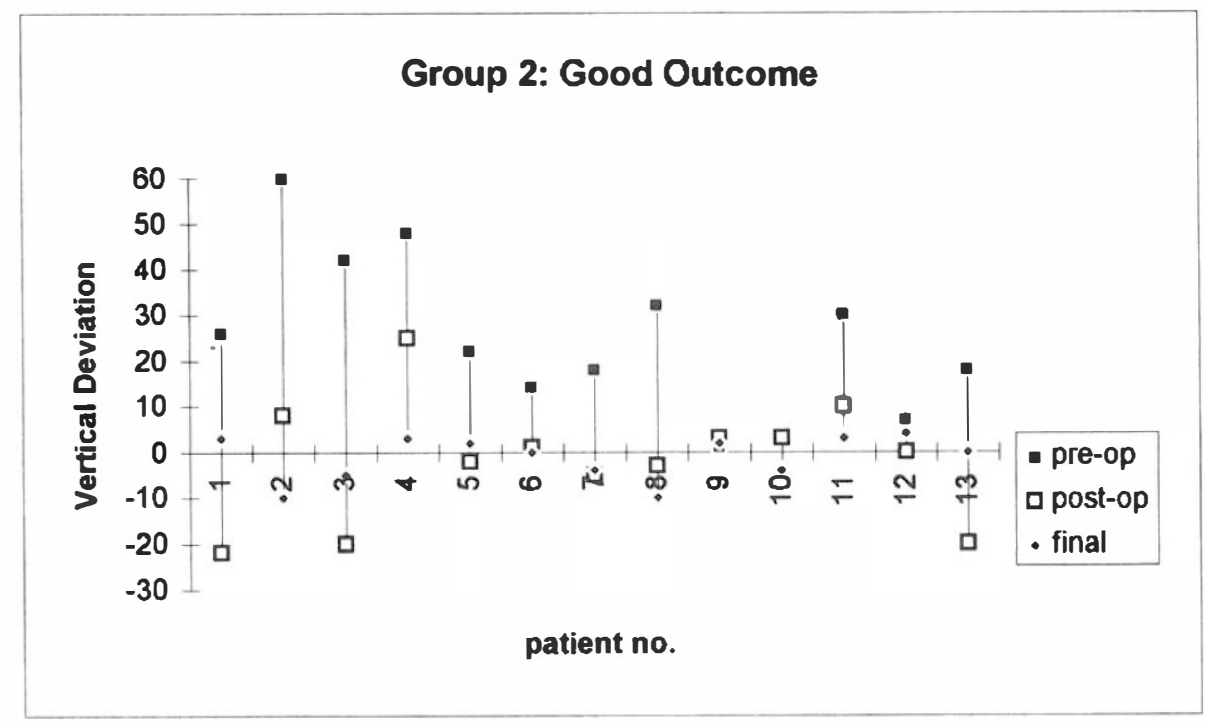

(a)

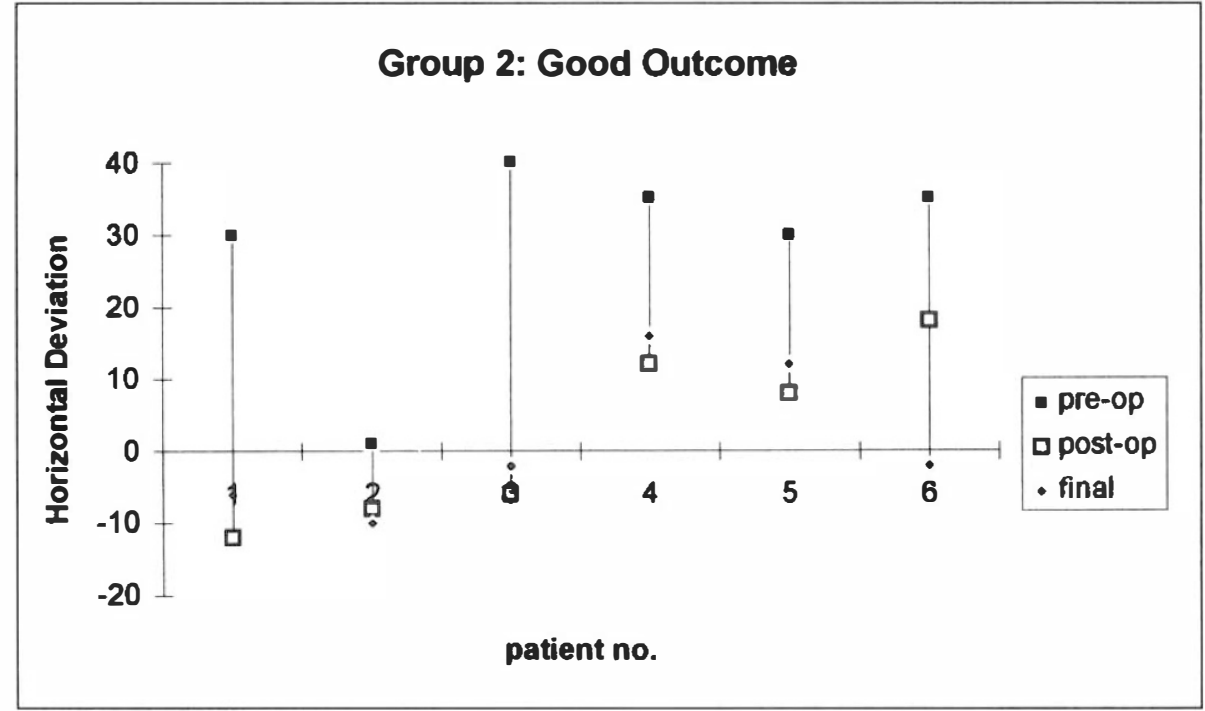

(b)

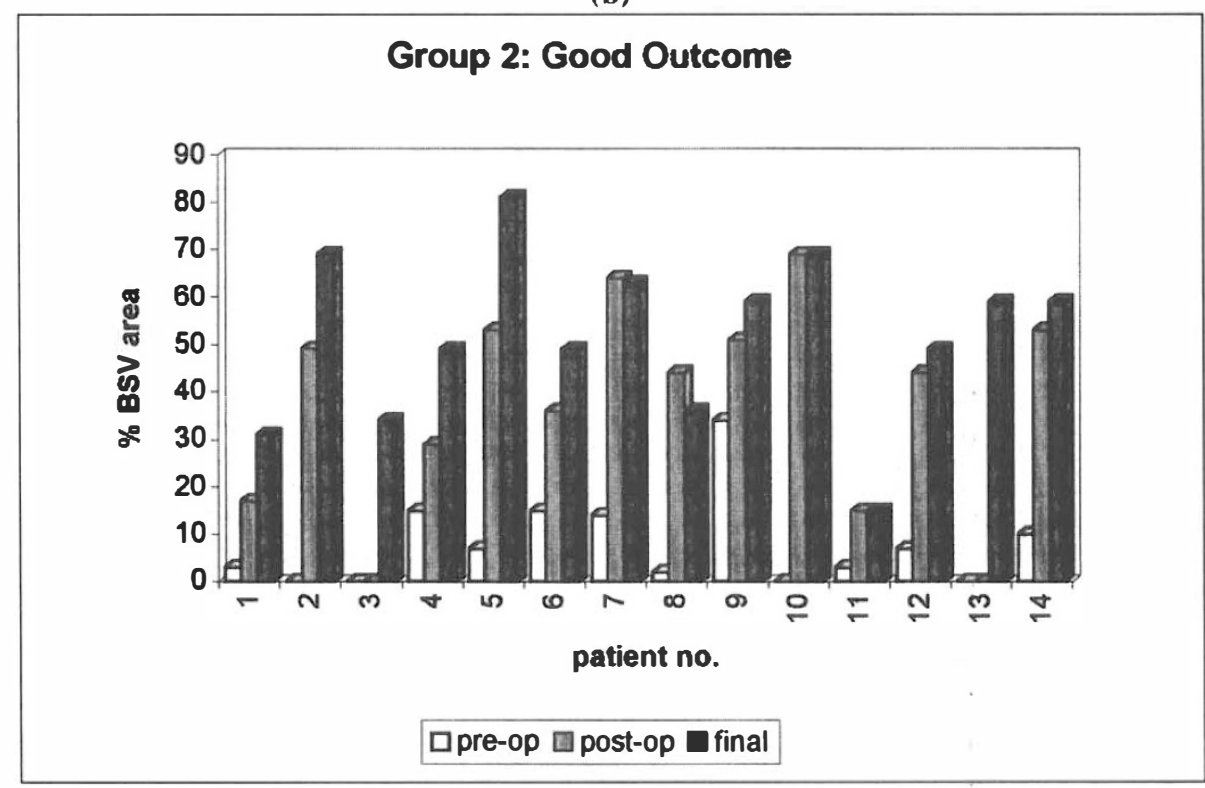

(c)

Fig. 6. Results of the good outcome group (group 2) showing the improvement in the vertical deviation, horizontal deviation and area of binocular single vision following surgery. 
and some were still on carbimazole. Both primary and secondary hypothyroidism were treated by $\mathrm{T}_{4}$. Fourteen patients had received oral prednisolone, 8 radiotherapy and 8 surgical decompression; 7 had had botulinum toxin injections.

Thirty-two patients had one strabismus operation, 8 had two operations and 1 had four operations. Of these operations 32 were vertical procedures, 16 combined vertical and horizontal, and 4 horizontal procedures (Fig. 5). Adjustable sutures were used in 47 operations but in 24 cases no adjustment was necessary and the suture was simply tied off. In all cases except 2 the tight muscles only

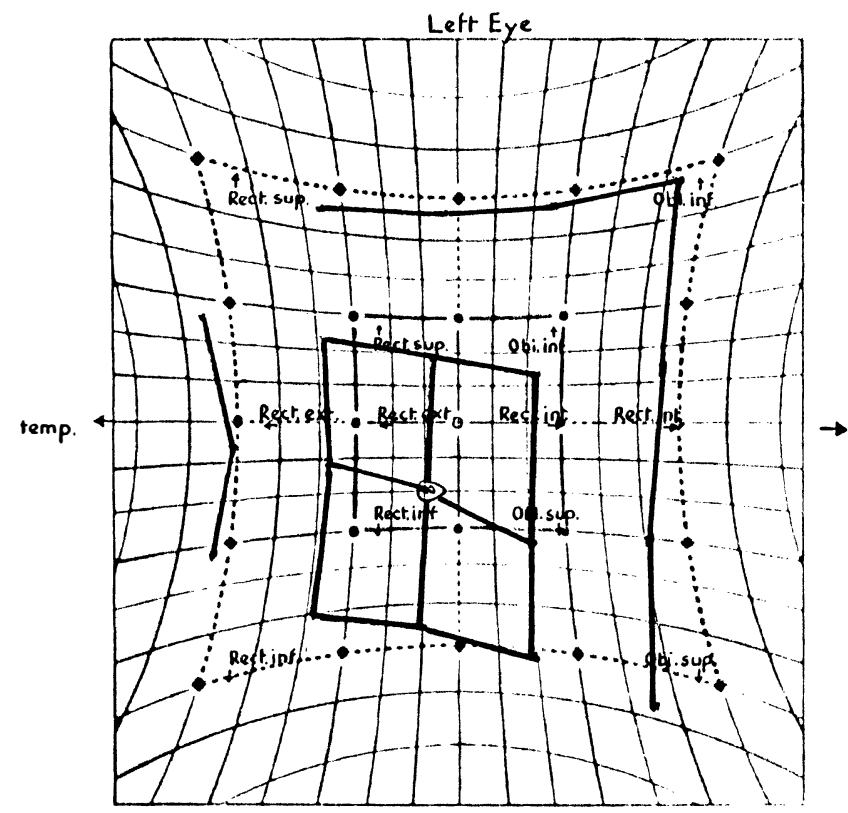

Green before left eye

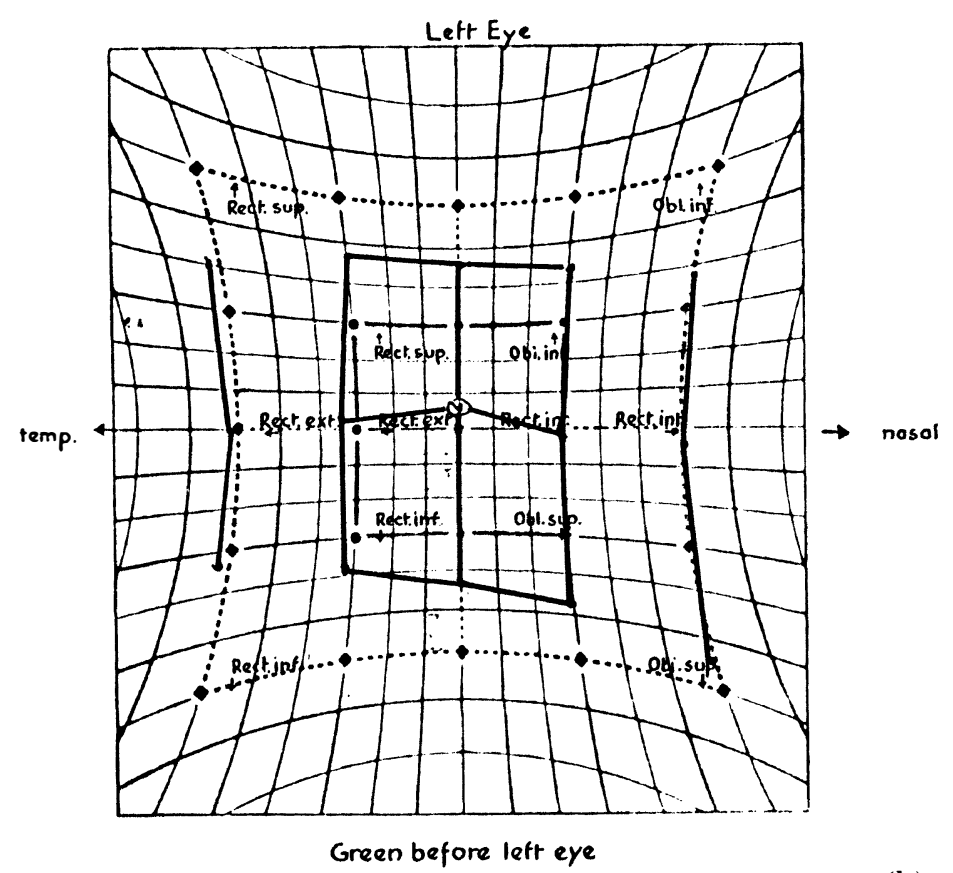

(b) were recessed: the inferior rectus in 34 procedures, medial rectus in 14 and the superior rectus in 9.

Twenty patients had excellent results of BSV, extending downwards more than $30^{\circ}$ without the help of prisms or a compensatory head posture. Fourteen patients had good results where the same area of BSV needed a small compensatory head posture or not more than 6 dioptres of prism (Fig. 6). Four satisfactory and 2 poor results showed smaller areas of BSV with worsening head postures and stronger prisms.

Muscle surgery in thyroid eye disease is almost unique in often requiring adjacent rectus muscles to be operated

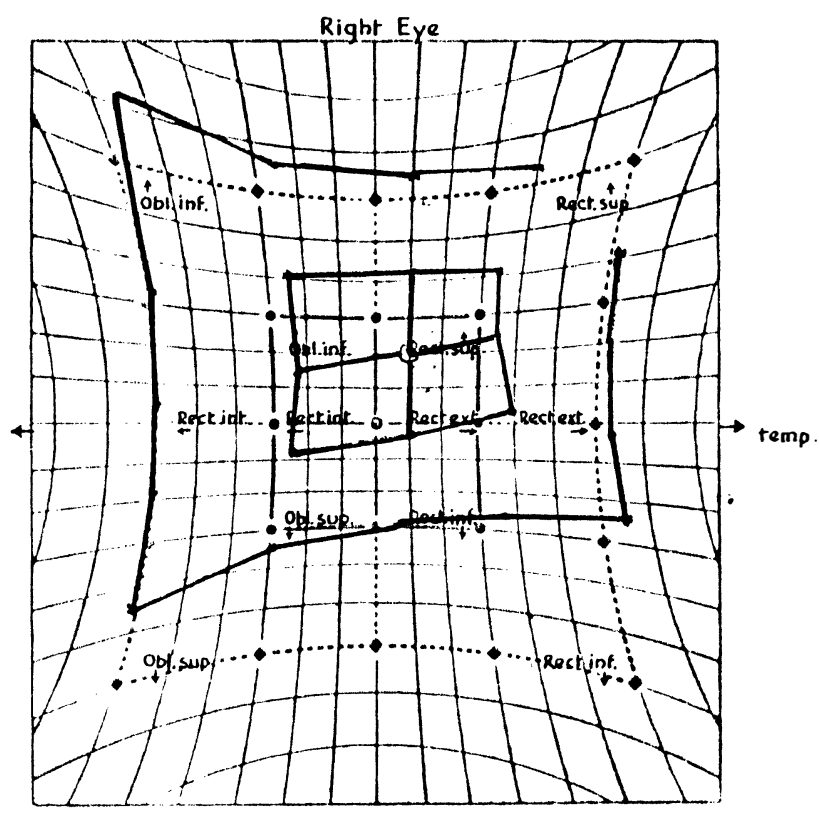

Green before right eye

(a)

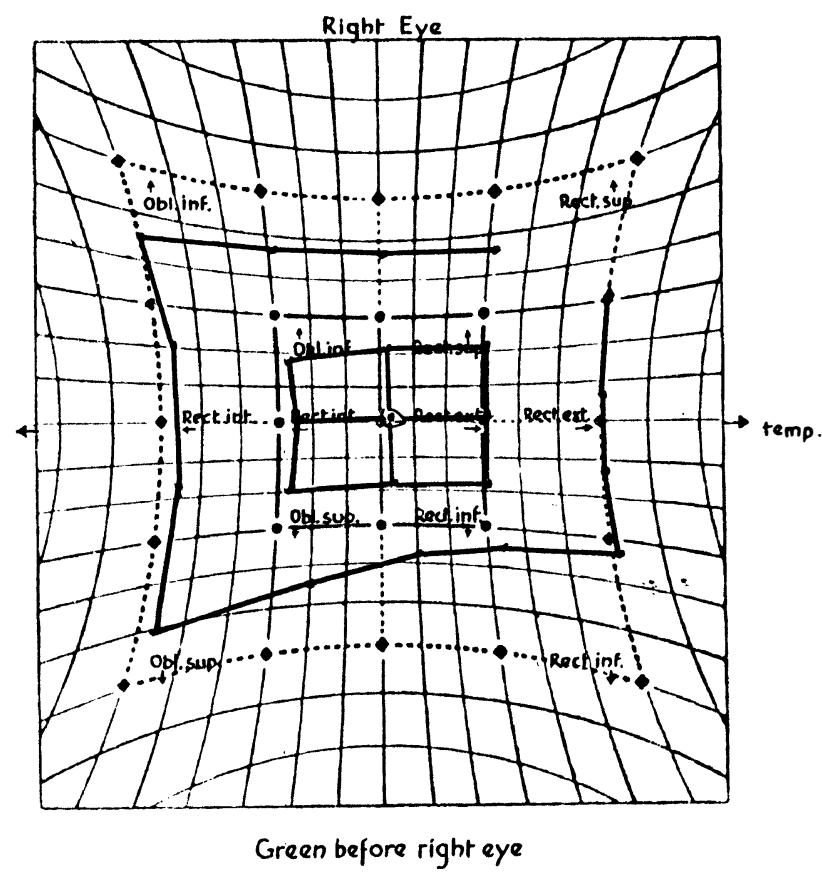

Fig. 7. Hess charts of patient 1 before (a) and 1 day after right superior rectus recession (b). (Continues). 
on at the same time, usually inferior rectus and medial rectus. In 1978 one of us (P.F.) first reported ${ }^{12}$ that this could cause anterior segment ischaemia in the lower nasal quadrant with slight pupillary irregularity and patchy loss of posterior iris pigment in this quadrant. Recently P.F. has, with Jane Olver, been using a vessel-sparing technique for recessions of (usually) the inferior rectus but leaving one or more of its anterior ciliary vessels intact. Naturally anatomical preservation has to have post-operative patency demonstrated by fluorescein angiography. Sometimes the muscles are so tight that the globe cannot be rotated into a position which permits vessel-sparing surgery.

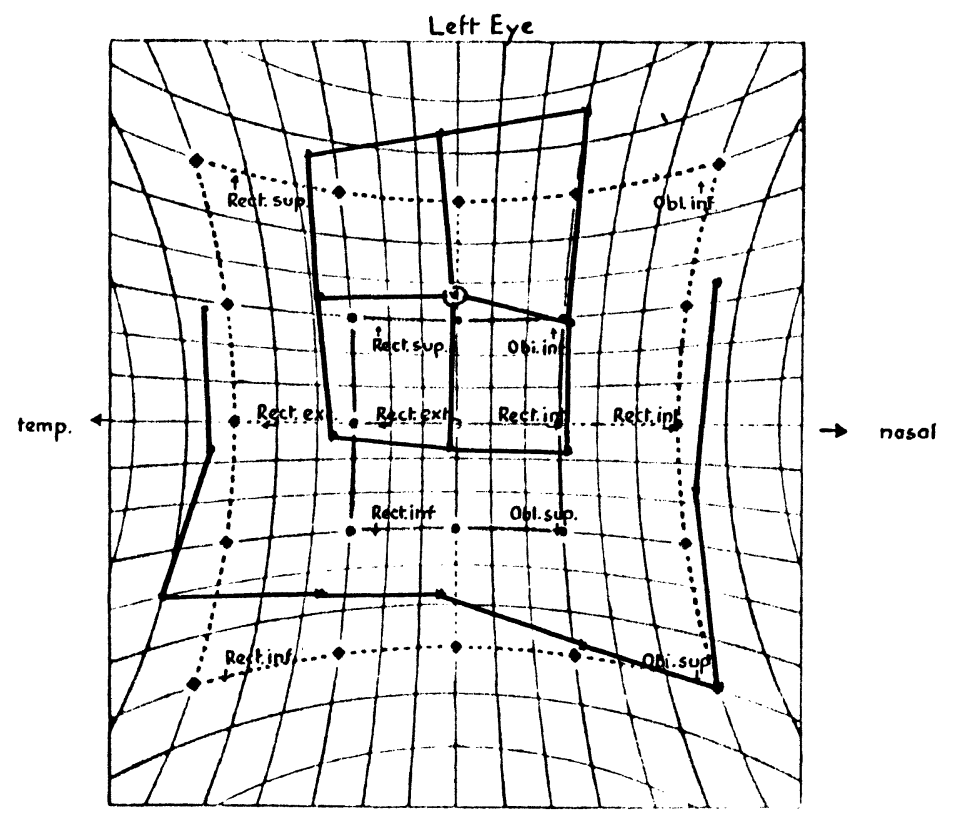

Green before left eye

(c)

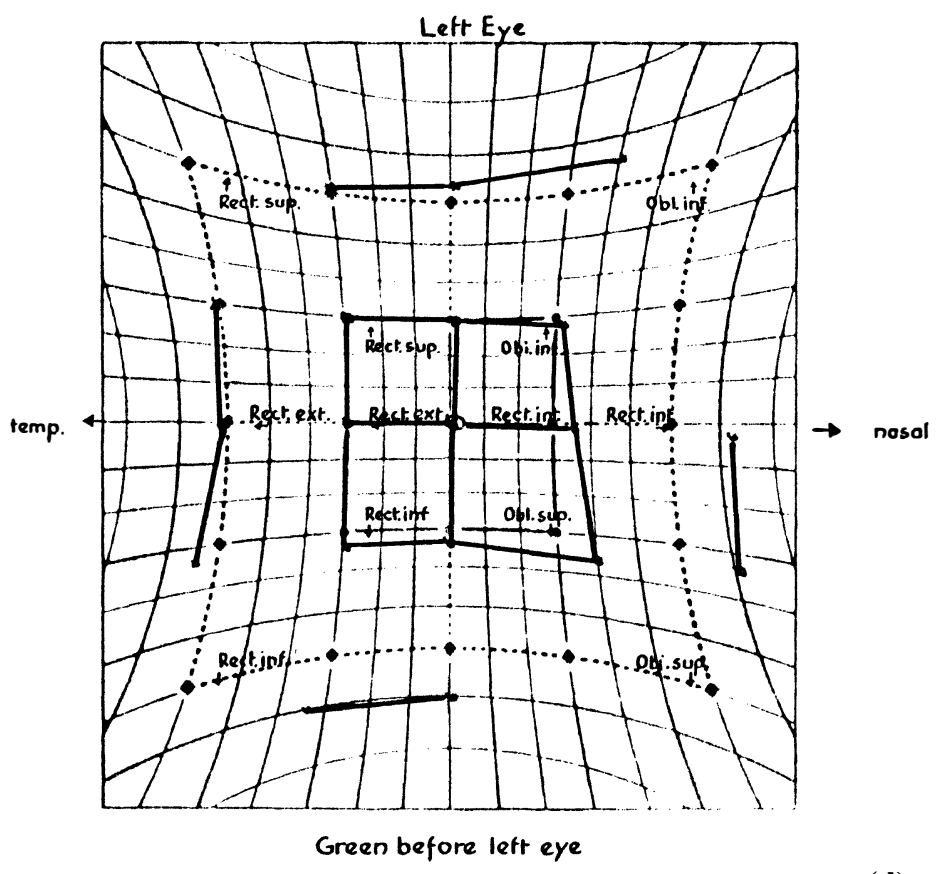

(d)
The powerful traction necessary to pull the globe into a position where muscle surgery can be performed can cause the intraocular pressure to rise to $50-70 \mathrm{mmHg}$. Such traction can be sustained safely for short periods only. Occasionally the tightness is such that it seems impossible even to insert sutures into the muscle. The temptation to do a free tenotomy should be resisted since this will invariably be followed by overcorrection. Perseverance virtually always allows recession to be done.

For the best long-term results a first day post-operative undercorrection of vertical deviation must be achieved. Patient 1 with right hypertropia of 22 prism dioptres

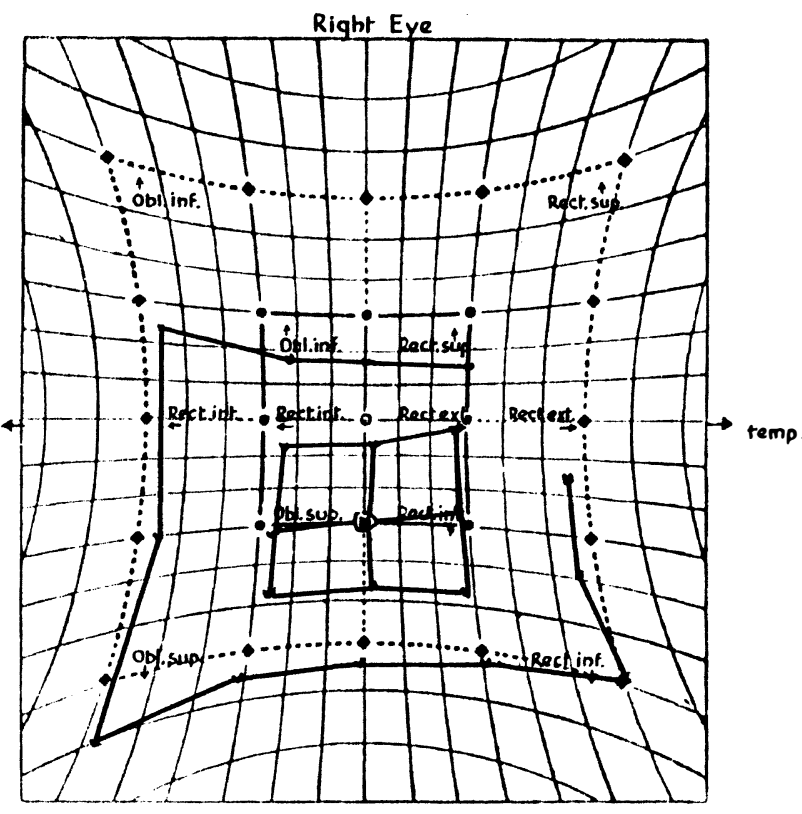

Green before right eye

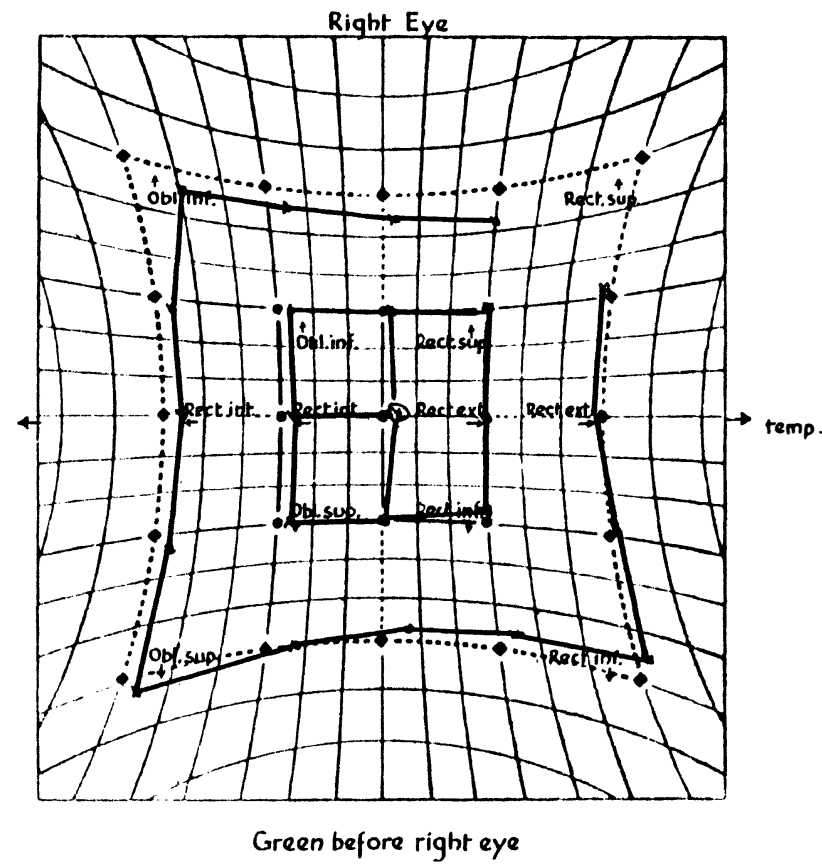

Fig. 7 (continued). Five months later the right hypertropia of 22 prism dioptres had overcorrected to 30 prism dioptres of left hypertropia $(c)$. Finally, right inferior rectus recession gave an excellent result $(d)$. 

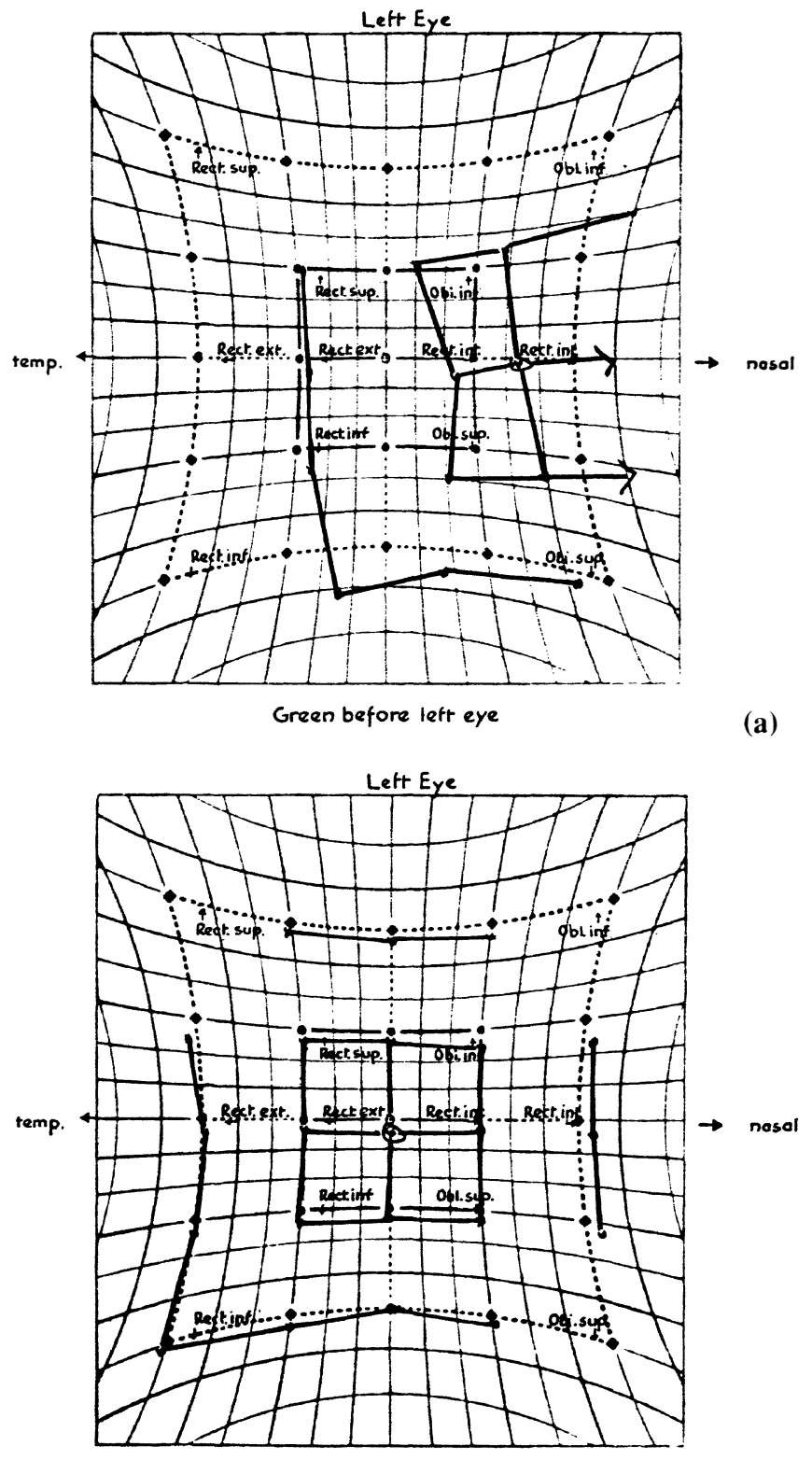

Green before lett eye

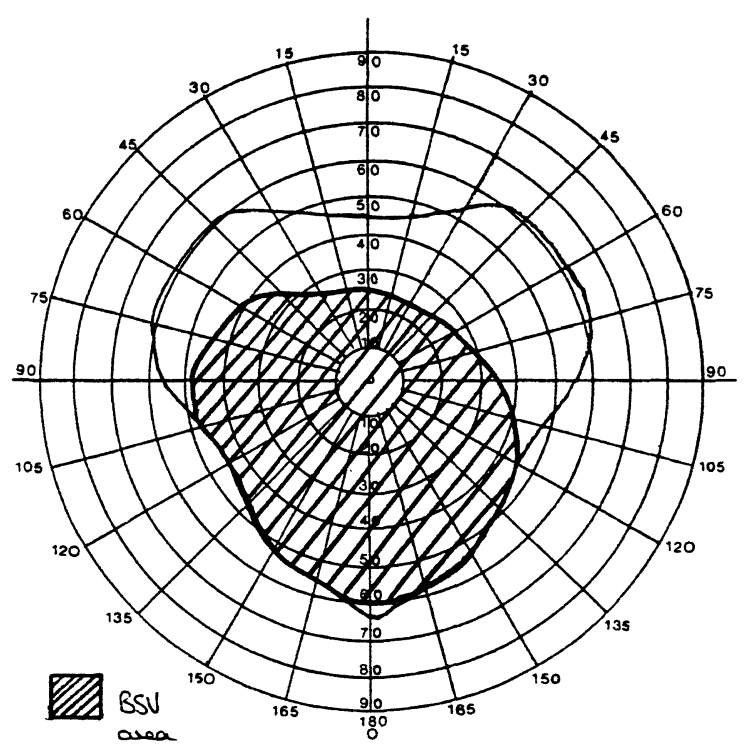

(c)

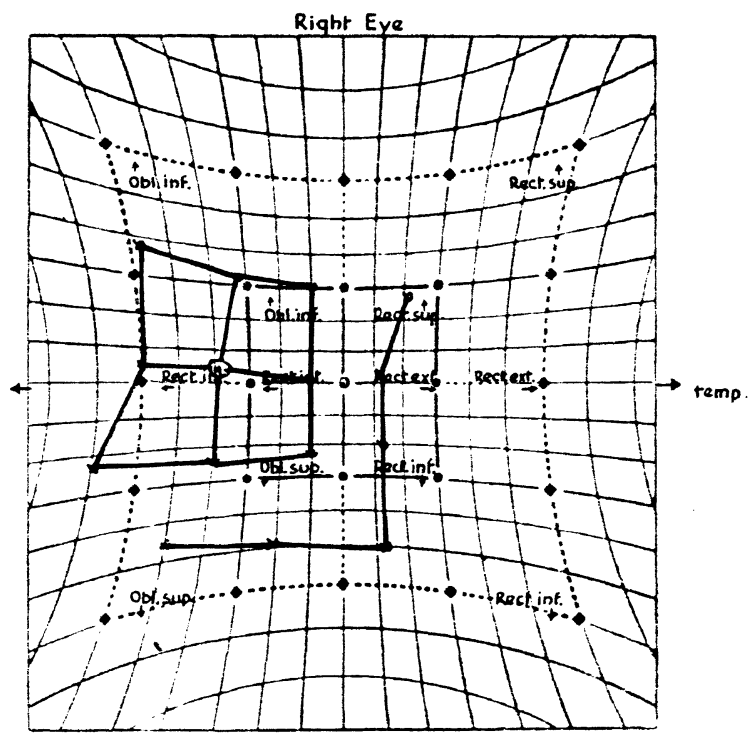

Green before right eye

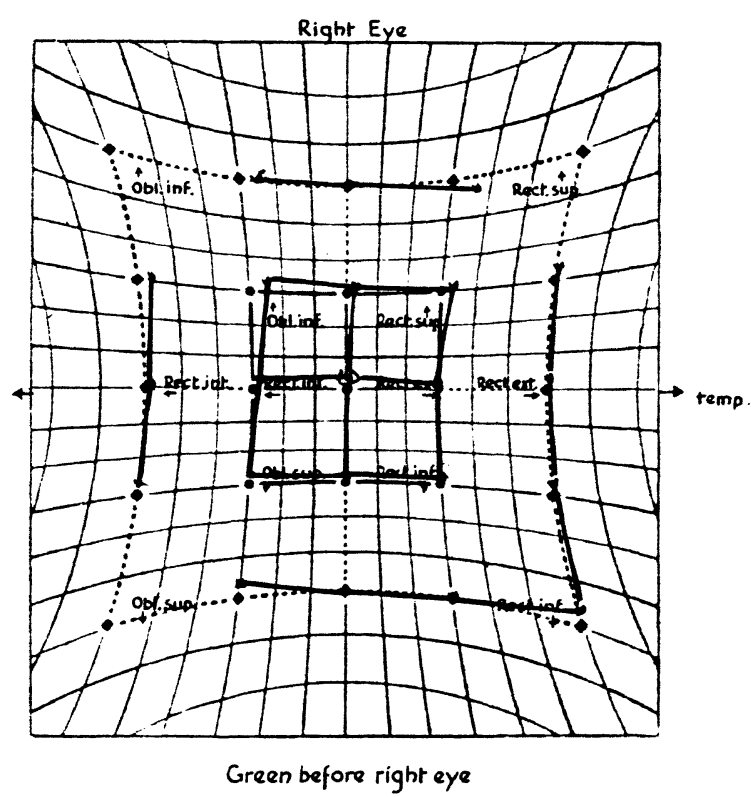

Fig. 8. Hess chart of patient 2 with a large esotropia and right hypertropia (a). After bilateral medial and inferior rectus recessions, using adjustable sutures on the tw'o muscles on the right side, she had an excellent result and regained a BSV field of $71 \%$ of normal $(b, c)$. 
(Fig. 7a) responded to a right superior rectus recession of $6 \mathrm{~mm}$ by being straight in the primary position (Fig. 7b). Two weeks later she had overcorrected to left hypertropia of 22 prism dioptres, increasing over 5 months to 30 prism dioptres (Fig. 7c). This responded well to right inferior rectus recession of $6 \mathrm{~mm}$, together with right medial rectus recession of $4 \mathrm{~mm}$ to give a final, lasting result of right hypertropia of 3 prism dioptres (Fig. 7d).

Patient 2 with 35 prism dioptres of esotropia and 8 prism dioptres of right hypertropia (Fig. 8a) underwent four muscle recessions at one operation. The right inferior rectus was recessed $6 \mathrm{~mm}$ and the right medial rectus 5 $\mathrm{mm}$, both on slides, and the left inferior rectus and left medial rectus were both recessed $4 \mathrm{~mm}$ using fixed sutures (Fig. 8b). From having no area of BSV the operation gave a field of BSV of $71 \%$ of normal (Fig. 8c).

One of the major complications of recessing very tight inferior recti is the risk of significant post-operative lower lid retraction measured as the height of sclera visible between the 6 o'clock limbus and the lid margin. Dissecting off the lower lid retractors from the inferior rectus muscle sheath as far back as possible, i.e. $15-16 \mathrm{~mm}$ from the inferior rectus insertion, helps to reduce but does not abolish this effect. Other surgeons are trying different methods but none is universally successful yet. Our figures for 36 inferior rectus recessions in 30 patients $(25$ procedures using adjustable sutures, 12 of which required adjusting), were as follows. The inferior rectus recessions were between 3 and $8 \mathrm{~mm}$. Dividing them into two groups of small inferior rectus recessions of 3-5 $\mathrm{mm}$ and large recessions of 5.5-8 $\mathrm{mm}$ the pre-operative lower lid retraction was $0.29 \mathrm{~mm}$ and $0.20 \mathrm{~mm}$ respectively. After surgery these figures were $0.86 \mathrm{~mm}$ (range $0-3 \mathrm{~mm}$ ) and $2.0 \mathrm{~mm}$ (range $0-4 \mathrm{~mm}$ ). This emphasises the importance of the correct order for surgery in thyroid eye disease, strabismus surgery to align the eyes first and finally lid surgery to match the lids to the globes.

The recommendations for successful surgery in this difficult condition are: make and maintain the patient euthyroid, have demonstrably stable ocular movements for at least 6 months, recess tight muscles using adjustable sutures and aim to undercorrect the vertical deviation.

The immunohistochemistry and electron microscopy were both carried out in Professor Susan Lightman's department at the
Institute of Ophthalmology. Dr Anastasia Pappa's research is supported by a grant from the Royal National Institute for the Blind. I am indebted as always to Jenny Rignold for preparing the manuscript.

Key words: Muscles, Surgical correction, Thyroid eye disease.

\section{REFERENCES}

1. Trokel SL, Jakobiec FA. Correlation of CT scanning and pathologic features of ophthalmic Graves' disease. Ophthalmology 1981;88:553-64.

2. Ossoinig KC. Ultrasonic diagnosis of Graves' ophthalmology. In: Gorman CA, Waller RR, Dyer JA, editors. The eye and orbit in thyroid disease. New York: Raven Press, 1984: 185-211.

3. Pappa, A, Fells P, Lightman S. Adhesion molecules in the immunopathogenesis of thyroid-associated ophthalmopathy. In: Kaufmann H, editor. Transactions of the XXth Meeting of the European Strabismological Association, Brussels 1992: 365-6.

4. Bahn RS, Heufelder AE. Orbital connective tissue in endocrine ophthalmopathy. In: Kahaly, G, editor. Endocrine ophthalmopathy: molecular, immunological and clinical aspects. Basel: Karger, 1993: 47-57.

5. Schuler M, Hansen C, Winand R, Staer C, Otto E, Beyer J, Kahaly G. Urinary and plasma glycosaminoglycans in endocrine ophthalmopathy. In: Kahaly G, editor. Endocrine ophthalmopathy: molecular, immunological and clinical aspects. Basel: Karger, 1993: 58-67.

6. Pappa A, Jackson P, Munro P, Fells P, Lightman S. Glycosaminoglycans in the pathogenesis of thyroid-associated ophthalmopathy. In: Kaufman H, editor. Transactions of the XXIst Meeting of the European Strabismological Association, Salzburg, 1993: 359-63.

7. Bahn RS, Heufelder AE. Pathogenesis of Graves' ophthalmopathy. N Engl J Med 1993;329:1468-75.

8. Lyons CJ, Vickers SF, Lee JP. Botulinum toxin therapy in dysthyroid strabismus. Eye 1990;4:538-40.

9. Simonsz HJ, Kommerell G. In Graves' disease, increased muscle tension and reduced elasticity of affected muscles is primarily caused by active muscle contraction. Neuro-Ophthalmol 1989;9:243-6.

10. Fells P. Thyroid-associated eye disease: clinical management. Lancet 1991;338:29-32.

11. Fells P, McCarry B, Aylward GW. Ocular muscle surgery in thyroid eye disease. Orbit 1992;11:169-75.

12. Fells P, Marsh RJ. Anterior segment ischaemia following surgery on two rectus muscles. In: Reinecke RD, editor. Strabismus. Proceedings of the third meeting of the International Strabismological Association, Kyoto. New York: Grune \& Stratton, 1978:375-80. 